\title{
1 Experimental design and statistical analyses of fish growth studies
}

2 Helgi Thorarensen ${ }^{\mathrm{a}^{*}}$, Godfrey Kawooya Kubiriza ${ }^{\mathrm{a}, \mathrm{b}, \mathrm{c}}$, Albert Kjartansson Imsland ${ }^{\mathrm{d}, \mathrm{e}}$

3 a Department of Aquaculture and Fish Biology, Hólar University College, 550 Sauðárkrókur,

4 Iceland

$5 \quad{ }^{\mathrm{b}}$ Department of Biological Sciences, College of Natural Sciences, Makerere University, P. O.

6 Box, 7062, Kampala, Uganda

7 ' United Nations University - Fisheries Training Programme, Skulagata 4, 101 Reykjavik,

$8 \quad$ Iceland

$9{ }^{\mathrm{d}}$ Department of Biology, University of Bergen, High Technology Centre, 5020 Bergen,

10 Norway

11 eAkvaplan-niva Iceland Office, Akralind 4, 201 Kópavogur, Iceland,

12 Keywords: growth studies; statistical power; minimum detectable difference; number of

13 replicates; sample size; ANOVA; polynomial; non-linear models

14 *Corresponding author. Tel: +354 455 6300; fax +354 4556301

15 E-mail address: helgi@ holar.is (H. Thorarensen)

16 


\section{Abstract}

Every year, numerous studies are published that compare the effects of different factors on the growth of aquaculture fish. However, comparatively little attention has been given to the experimental designs of these studies - in how many rearing units should each treatment be replicated, how many fish should be in each tank $(n)$ and how should the data be analysed. The reliability of the results increases with increased replication and $n$. In reality, however, the experimental design must strike a balance between limited resources and the reliability of the statistical analysis. A survey of recent publications in Aquaculture suggests, that most $(83 \%)$ aquaculture growth studies apply each treatment in triplicates with an average of 26 fish in each tank (range: 4 to 100). The minimum difference that can reliably be detected with statistical analyses is determined by the number of replications of each treatment, $n$, the variance of the data and the number of treatments applied. In the present study, we accumulated information on the variance of data in aquaculture growth studies on different species to estimate the minimum detectable difference and to assist researchers in designing experiments effectively. These results suggest that the variance is similar for different aquaculture species and, therefore, the same designs (level of replication and $n$ ) are suitable for studies on different species of fish.

The minimum difference (MDD) in mean body-mass of different treatment groups that can be detected in a typical aquaculture study (triplicates, 25 fish in each tank and average variance) with $80 \%$ statistical power (less than $20 \%$ chance of Type II error) is around $26 \%$ of the grand mean. Increasing the $n$ from 25 to 100 will reduce the MDD to $19 \%$ of the grand mean, while a further increase in $n$ will have comparatively lesser effect. Increasing replication to quadruplicates or sextuplicates (with $n$ as 100), will further reduce the MDD to $16 \%$ and $12 \%$ of the grand mean respectively. MDD under $10 \%$ of the grand mean is only possible when fish for the experiment are selected within a narrow size range to reduce variance. 
43 Simulations were performed, where samples (experiments) were repeatedly drawn from 44 artificial populations with identical distribution and with the same experimental design as is commonly used in growth studies. Two of the populations had dose-dependent responses to treatment while one population showed no response to treatment. The resulting data was analysed with a mixed model ANOVA and by fitting either polynomials or asymptotic models to the data. Contrary to earlier suggestions, the critical treatment (minimum treatment to generate maximum response) estimated with the ANOVA approached more closely the population responses than did the critical treatments estimated with the non-linear models.

Keywords: Growth studies, statistical power, minimum detectable difference, number of replicates, sample size, ANOVA, polynomial, non-linear models 


\section{Introduction}

Information on the effect of feed ingredients, physical environment and other factors on the growth of fish are important for the development of aquaculture. Therefore, growth studies are common in aquaculture research where the mean sizes of different groups are compared following various treatments; the objective being to predict the performance of populations (all fish of the same species/strain) under different conditions.

The design of aquaculture growth experiments usually includes replication of treatments in two or more rearing units (e.g. tanks, ponds or net pens) where the replicates are considered independent samples from the populations. How accurately the results of experiments reflect the mean responses of the populations depends primarily on the number of fish sampled (within each replicated unit), the number of replicates and the variance of responses, both among individual fish within a replicated unit and among replicates.

A number of approaches have been used to analyse the results of growth studies, but the method most commonly used is analysis of variance (ANOVA). A cursory examination of growth studies (Table 3) published during the last year in the journal Aquaculture (29 in total) suggests that ANOVA is used in in some capacity in all studies although $24 \%$ of the studies complement the analysis of dose response data with linear or non-linear methods.

In growth studies where treatments are replicated, individual fish should not be considered the experimental units. The fish within a tank are all exposed to the same "tank effects" (differences between tanks independent of treatment effects) and complicated interactions among the fish may contribute to variability within the tank that are not caused by the treatment (Gardeur et al. 2001; Imsland 2001; Koslow \& Hurlbert 2006). In fact, it can be argued that because of the common "tank effect", individual fish within a tank are not independent samples from the population but are instead "pseudoreplicates" as defined by Hurlbert (1984). A better approach is to perform ANOVA based on the total biomass or mean 
body-mass in each tank (Cowey 1992; Smart et al., 1998) or, better still, to use a mixed model ANOVA where treatments are fixed factors and tanks are nested as random factors within treatments. With the latter method, the information on individual fish is modelled to fully account for the data structure (Ruohonen 1998, Ling and Cotter 2003). If the design of the experiment is balanced, i.e. the number of fish in all tanks and the number of tanks in all treatments is the same, the results of the simple and mixed model ANOVA will be the same. However, in long term growth studies the design may not be balanced, since mortality can vary among rearing units and all fish from single rearing units may be lost due to mishaps. When the design is not balanced, a mixed model should be used since the risk of type I error (rejecting a correct hypothesis) is increased when a simple ANOVA is used for the analysis of unbalanced data (Ruohonen 1998).

In recent years, methods for mixed model analysis have developed rapidly and now many software packages such as SAS (SAS Institute Inc., Cary, NC, USA) and R (R Core Team 2014) offer the possibility of linear mixed models with the Kenward-Roger modification of $F$ tests (Kenward and Roger, 1997, 2009). The Kenward-Roger modification adjusts the $F$ values and degrees of freedom depending on the size of the "tank effect" and thus increases statistical power when the "tank effect" is small. The method has been used in aquaculture growth studies (Tobin et al. 2006; Schram et al. 2012). Over $83 \%$ of the growth studies published last year in Aquaculture use the mean body-mass or total biomass in each tank as the unit of analysis while only $11 \%$ used a mixed model analysis (Table 3).

In ANOVA, the null hypothesis of no effect of experimental treatments is tested and the means of the treatment groups are considered significantly different when the test statistics $(p$ value) indicates that the probability of the null hypothesis being true is less than $5 \%(\alpha$ level less than 0.05 ). In other words, the probability of rejecting a correct null hypothesis (type I 
error) is less than 5\%. However, it is also possible that an incorrect hypothesis is not rejected

106 and differences among means are not detected where they truly exist. Failing to reject an 107 incorrect hypothesis is called Type II error. The probability of Type II error is $\beta$ and the 108 power of a statistical test is defined as $1-\beta$. There is no conventional criterion for statistical 109 power as there is for $\alpha$, although a minimum of $80 \%$ is commonly regarded as suitable 110 (Araujo \& Frøyland 2007). Statistical power is rarely reported in aquaculture growth studies 111 (Searcy-Bernal 1994) indicating that researchers are less concerned with Type II error than 112 they are with $\alpha$ and Type I error.

113 The statistical power of mixed models depends on five factors: (1) The difference among 114 means caused by the treatment (effect size), (2) the variance of the data, both among fish 115 within a tank and among tanks receiving identical treatments, (3) the number of replicate 116 tanks, (4) the number of fish within each tank and (5) the number of treatments tested (Ling 117 and Cotter 2003, Sokal and Rolf 2012). Statistical power increases with increased effect size, 118 the number of replicate tanks and the number of fish within each replicate tank while 119 statistical power is reduced with increased variance and number of treatments tested (Ling and 120 Cotter 2003). Hence, to secure acceptable statistical power, replications and sample size per 121 replicate should be maximized. However, the number of tanks available and the cost of 122 resources for aquaculture growth studies are usually limited. Therefore, experimental design must strike a balance between acceptable power and the available resources.

124 The issue of the minimum detectable difference (MDD) in aquaculture studies, i.e. the 125 minimum difference that is likely to be detected with $80 \%$ statistical power, has received little 126 attention. Ling and Cotter (2003) shed important light on this subject when they compiled 127 information on the coefficient of variation within tanks $\left(C V_{\varepsilon}\right)$ and the coefficient of variation 128 among tanks within treatment $\left(C V_{\beta}\right)$ for triploid Atlantic salmon. In the present study, we compiled information on variance in body-mass in growth studies on different fish species to 
130 be able to estimate statistical power and the MDD. This information was then used to

131 calculate the expected statistical power and effect size for experimental designs with different

132 levels of replication and number of fish in each replicate tank.

133 Dose-response designs, where treatments are applied at incrementing levels of e.g. nutrient

134 content or water quality, are common in aquaculture growth studies. These data can be

135 analysed either with ANOVA or by using different linear and non-linear methods. The latter

136 include: Broken line analyses, where two straight lines are fitted to the data, polynomial

137 regression or non-linear regression models that fit asymptotic curves to the data (Baker 1986,

138 Cowey 1992, Shearer 2000). When the results are analysed with ANOVA, the critical

139 response is usually determined as the lowest treatment level that gives a response that is not

140 significantly different from the maximum response. However, this approach has been

141 criticised by Baker (1986) and then later by Cowey (1992) and Shearer (2000). After

142 reviewing a number of published growth studies with dose-dependent relationship, Shearer

143 (2000) concluded that ANOVA may underestimate the critical treatment level by as much as

$14450 \%$ due to the inability of the method to detect small differences. Instead several authors

145 (Baker 1986, Cowey 1992, Shearer 2000) recommend the use of linear or non-linear methods

146 and suggested that they provided more accurate results. However, fitting lines of different

147 shape assumes that there is a certain underlying structure to the data. Moreover, due to the

148 inherent variability in aquaculture growth data it may be difficult to determine visually if the

149 response is polynomial or asymptotic. Therefore, it is questionable if this approach is more

150 appropriate than ANOVA. A second objective of this study was to use simulation studies to

151 compare the fidelity of different methods of statistical analysis to the true underlying

152 responses of populations and the conclusions drawn based on their results. 
154

155

156

157

158

159 Data on Arctic

159 Data on Arctic

161

162

163

164

165

166

167

168

169

170

171

172 treatments $\left(\hat{\sigma}_{\beta}^{2}\right)$ was calculated as $\hat{\sigma}_{\beta}^{2}=\frac{\left(M S_{\text {within }}\right)-\hat{\sigma}_{\varepsilon}^{2}}{n}$, where $n$ is the number of fish in each

173 tank. The coefficient of variation for tanks within treatments $\left(C V_{\beta}\right)$ was calculated

174 as $C V_{\beta}=\frac{\hat{\sigma}_{\beta}}{\bar{X}}$. The statistical power was estimated as described by Ling and Cotter (2003).

175 Briefly, the mean variance of treatment groups $\left(s_{\bar{Y}}^{2}\right)$ was estimated as: $s_{\bar{Y}}^{2}=\frac{M S_{\text {within }}}{n b}$, where $b$ 
176 is the number of replicate tanks within treatments. The $s_{\bar{Y}}^{2}$ was used to compute Tang's

177 parameter $(\phi)\left(\right.$ Tang, 1938) as $\phi=\sqrt{\frac{d^{2}}{2 a s_{\bar{Y}}^{2}}}$; where $d$ is the difference between means and $a$ is

178 the number of treatments tested. This value was then used to compute the non-centrality

179 parameter $(\lambda)$ as: $\lambda=a \phi^{2}$.

180 The statistical power of each study was then calculated with the program G*Power (Faul et

181 al., 2007) using the $\lambda$ and degrees of freedom with the $\alpha$-level set at 0.05 . This protocol was

182 repeated to model the MDD for different values of $C V_{\varepsilon}$ and $C V_{\beta}$ (Table 2) using levels of

183 replications $(b)$ from 2 to 6 and number of fish in each tank $(n)$ from 10 to 1000.

\section{2.3. Simulation studies}

185 Simulations were performed to compare three different methods for statistical analysis of

186 growth studies with a graded response: ANOVA, a second order polynomial and a three 187 parameter logistic growth model. The simulations were performed with $\mathrm{R}$ ( $\mathrm{R}$ Core Team 188 2014). The datasets used for the analysis represent random samples from three different 189 populations:

190 Res45\%: A population with a saturation type relationship to treatment where the 191 response increased with treatment level until it plateaued with a response of $100 \%$ at 192 treatment levels over $100 \%$. The response to the minimum treatment was $45 \%$ lower 193 than the maximum response (100\%) (Fig. 1).

194 Res 11\%: A population with saturation type relationship to treatment where the minimum response was $11 \%$ lower than the maximum response. The maximum response was $100 \%$ and reached when the treatment level was $100 \%$ (Fig. 1). Res0\%: A population with no response to treatment (Fig. 1). 
198 The population responses to the treatments were normally distributed at each treatment level

199 and the same variance was assumed for all responses regardless of treatment level.

200 The simulations were performed on 1000 datasets generated from each population. The 201 simulations were made for experiments with 18 tanks and 50 fish in each tank. The datasets were random samples, generated based on the mean responses of the population at different treatment levels with equal variance for the means of tanks within all treatment levels. The means of tanks within treatments were normally distributed with a standard deviation equal to $4.5 \%$ of the grand mean for tanks within treatments. The residual variance within each tank was normally distributed with a standard deviation equal to $30.6 \%$ of the grand mean. These standard deviations are the same as the mean $C V_{\beta}$ and $C V_{\varepsilon}$ for all species found in this study (Table 1). In the data sets generated, the treatment levels tested were in arbitrary units expressed in percentages and could range between $85 \%$ and $121 \%$. To reflect the strengths of different statistical approaches, tanks were allocated differently for mixed model ANOVA, 211 polynomial models and non-linear models. In the mixed model simulations, six levels of treatments were tested, each in triplicate. In each sample, the lowest treatment levels tested ranged at random between $85 \%$ and $90 \%$ and then successive treatment levels were applied in

$2145 \%$ increments. The samples for the polynomial and non-linear models were in duplicate at 215 nine treatment levels. In each sample, the lowest treatment level tested ranged at random 216 between $85 \%$ and $89 \%$ and then successive treatment levels were applied in $4 \%$ increments 217 covering a range of treatment levels of $32 \%$.

218 Three methods were used to analyse the data:

219 1) Mixed model ANOVA with tanks as random factors nested within treatments and measurements of individual fish in each tank using the lme function within the nlme package (Pinheiro et al. 2014) in R. All designs were balanced with the treatment 
degrees of freedom as 5 (treatment levels - 1) and the residual degrees of freedom as 12 (treatment levels $\times($ tanks within treatments -1$))$.

2) Second order polynomial using the $\operatorname{lm}$ function in $R$.

3) Non-linear three parameter logistic growth model using a self-starting logistic function in R (SSlogis)

Three approaches were used to compare the analysis methods:

1. The critical treatment levels, the minimum treatment level required to generate a maximum response were estimated for all the models:

a. For the ANOVA, the highest treatment level that did not generate a response significantly different from those of the two highest treatment levels.

b. For the polynomial model, the critical level was the estimated treatment level that caused the maximum response.

c. In the logistic growth model, the treatment level causing a response that was $98 \%$ of the asymptote was arbitrarily chosen as the critical treatment. response and $Y$ is the population response.

3. The maximum responses, estimated from the predicted values of the ANOVA and the second order polynomial and for the asymptote of the logistic regression model. values: $\frac{1}{t} \sum_{i=1}^{t}\left(\hat{Y}_{i}-Y_{i}\right)^{2}$ where $t$ are the treatment levels tested, $\hat{Y}$ is the predicted 


\section{Results}

\subsection{Coefficient of variation for fish within tanks $\left(C V_{\varepsilon}\right)$}

244 In most studies, $C V_{\varepsilon}$ increased as the experiments progressed but tended to stabilize when the 245 factorial increase in body mass (mean body-mass / mean initial body-mass) was about 1.5

246 (Fig. 2a,b,c). However, this pattern was not entirely consistent: In the study on Atlantic 247 halibut, the $C V_{\varepsilon}$ was nearly constant throughout and in the study on tilapia the $C V_{\varepsilon}$ increased 248 progressively (Fig. 1a). At the end of the experiments, the mean $C V_{\varepsilon}$ was $30.6 \pm 4.5 \%$ (mean $249 \pm \mathrm{SD}$ ) and ranged from $15 \%$ to $56 \%$ (Table 1). There were no clear differences in final $C V_{\varepsilon}$ 250 for different species and the $C V_{\varepsilon}$ varied between different studies on a single species. Thus the final $C V_{\varepsilon}$ for Atlantic cod ranged from 32 to $56 \%$ (Fig. 2b; Table 1) and from 15 to $39 \%$ for Arctic charr (Fig. 2c; Table 1).

\subsection{Coefficient of variation for tanks within treatments $\left(C V_{\beta}\right)$}

The mean $C V_{\beta}$ at the end of all studies was $4.5 \pm 0.4 \%$ (Mean $\pm \mathrm{SD}$; range: $0-12$ ). The $C V_{\beta}$ increased initially in many studies but stabilised as the experiments progressed (Fig. 3a,c). However, this pattern was not consistent in all studies and in some, the $C V_{\beta}$ decreased as the experiments progressed (Fig. 3a,b). Of the 24 studies investigated, eight had a final $C V_{\beta}$ of zero; five had $C V_{\beta}$ ranging from $2 \%$ to $5 \%$, while 11 had $C V_{\beta}$ of above $5 \%$, the highest being $11 \%$ (Table 1).

\subsection{Correlation between initial and final $C V$ and body mass.}

In 20 studies (Table 1), information was available on both initial and final variance in bodymass. The final $C V_{\varepsilon}$ in these studies was significantly correlated with initial $C V_{\varepsilon}(\mathrm{r}=0.621$; $p<0.003 ; N=20$ ). Similarly, final $C V_{\beta}$ in different studies was significantly correlated with the initial $C V_{\beta}(\mathrm{r}=0.657 ; p<0.002 ; N=20)$. 
Information was available from several studies on Arctic charr and Atlantic cod (Table 1.). These data were used to compare the variance in studies on the two species. The final $C V_{\varepsilon}$ and $C V_{\beta}$ in experiments on both species $(P<0.05)$ - decreased with increasing final body mass (Fig. 4a, b). Adjusting for body mass, $C V_{\varepsilon}$ was significantly lower $(P<0.0001)$ in Arctic charr than in Atlantic cod (Fig4a); while $C V_{\beta}$ were not significantly different (Fig. 4b). However, the initial $C V_{\varepsilon}$ in the studies on Atlantic cod were higher than in the studies on Arctic charr and, when the initial $C V_{\varepsilon}$ is included as a variable in the model, the difference between the species was no longer significant.

\subsection{Statistical power and minimum detectable difference with $80 \%$ statistical power.}

When experiments are designed it is recommended that statistical power is $80 \%$. In the experiments analysed (Table 1), the mean statistical power estimated post hoc was $53.9 \pm 0.3 \%$ $($ mean \pm SD) and ranged from $12 \%$ to $100 \%$. The MDD was $18.1 \pm 12.8 \%$ (range: $4 \%$ to $56 \%$ ) of the grand mean.

To show how experimental design is likely to affect the MDD, we modelled MDD using different number of replications and numbers of fish within each tank. The MDD was modelled for medium, high or low $C V_{\varepsilon}$ and $C V_{\beta}$ using the average, maximum and minimum $C V_{\varepsilon}$ and $C V_{\beta}$ encountered (Table 1). For the purpose of the modelling, it was assumed that five different treatments were being tested.

The level of replication and the number of fish in each tank affects the MDD (Fig. 5a,b,c). For all levels of replication, the MDD decreases markedly with increasing $n$ until it reaches about 100. There is comparatively little gained in reduced MDD by increasing $n$ over 100 . For average $C V_{\varepsilon}$ and $C V_{\beta}$, designs in triplicate are required for reaching an MDD of $20 \%$ or less. 
Similarly, four to six replications can give a MDD of 10-14\% (Fig. 5a). A MDD under 10\% is 291 only possible when both $C V_{\varepsilon}$ and $C V_{\beta}$ are low (Fig. 6c); reaching 4 to $10 \%$ when $n$ is 100 .

\subsection{Comparison of different methods to analyse graded treatment growth data}

294

295

Datasets were generated from random samplings of three different populations (Fig. 1) based on the average $C V_{\varepsilon}$ and $C V_{\beta}$ (Table 1). In total, 1000 datasets were generated for each population and analysed using a mixed model ANOVA, a second order polynomial and logistic regression. The logistic regression failed to converge on average in $0.1 \%, 20 \%$ and $67 \%$ of trials for the Res $45 \%$, Res $11 \%$ and Res $0 \%$ populations respectively.

With the ANOVA, the estimated mean treatment level required to create a $100 \%$ response for the Res $45 \%$ population was $99.7 \%$, matching closely the critical treatment of the population (100\%) with $95 \%$ of estimated values being between $96 \%$ and $104 \%$ (Table 2). The second order polynomial overestimated the critical treatment of the population with more than $95 \%$ of the estimates being higher than $107 \%$ (Table 2). The critical treatment estimated through the logistic regression (Table 2) was 101\% (95\% range 97\%-107\%). However, it should be stressed that the critical treatment was arbitrarily chosen to be where the response reached $98 \%$ of the estimated maximum. Obviously the response level chosen will affect the estimate of the critical treatment value.

Analysis of the Res11\% population showed a significant treatment effect in $36 \%$ of tests with ANOVA and $51 \%$ with the polynomial tests. The mean critical treatment estimate from the ANOVA was 95\% (Range 90\%-103\%) while statistical analysis with the polynomial estimated the critical treatment values as 109\% (range: 102\%-113\%) (Table 2). The mean critical treatment estimate from the logistic regression was 103\% (range: 93\%-113\%). For the Res0\% population, where treatment had no effect (all responses were 100\%), the polynomial showed significant effects in $5 \%$ of tests while the mixed model ANOVA only 
315 showed significant differences in $1 \%$ of the analyses. As described above, the logistic 316 regression analysis did not converge in most of the analyses of samples from of the $0 \%$ 317 population.

318 The estimated maximum responses were similar for all methods of analysis with the 95\%

319 range of responses covering the population maximum response of $100 \%$. For the Res $45 \%$ 320 population, estimates from all statistical methods show a similar mean square residual 321 deviation from the population response (Table 2), while at Res $11 \%$ and Res0\% the residual 322 values for the ANOVA were slightly higher than for either the polynomial or the logistic 323 regression. The mean MDD in the ANOVA was $18.3 \%$ and $13.1 \%$ for the Res $45 \%$ and 324 Res11\% populations respectively.

\section{Discussion}

327 This is the first study to evaluate the variance, statistical power and MDD in growth studies of 328 various aquaculture species. Earlier, Ling and Cotter (2003) evaluated the variance in growth 329 studies of triploid Atlantic salmon, finding a mean $C V_{\varepsilon}$ of $28 \pm 8.6 \%$ (range: $14-41 \%$ ) and $330 C V_{\beta}$ of $3.2 \pm 1.9 \%$ (range: $1-7 \%$ ). In 29 growth studies on 24 species published during the last 331 year in Aquaculture (Table 3), the estimated mean $C V_{\beta}$ was 5\% (range: 0-49\%) while the 332 mean $C V_{\varepsilon}$, was $28 \%$. All these values are in accord with the results of the present study where 333 the $C V_{\varepsilon}$ and $C V_{\beta}$ (mean $\pm \mathrm{SD}$ ) were $30.6 \pm 4.5 \%$ (range: $15 \%-56 \%$ ) and $4.5 \pm 0.4 \%$ (range: 0 $33412 \%$ ) respectively. Both the present study and that of Ling and Cotter (2003), show that $C V_{\varepsilon}$ 335 and $C V_{\beta}$ for a single species can range widely among different studies. The only indication of 336 species differences in variance in body mass is the apparent difference in $C V_{\varepsilon}$ between the 337 Atlandic cod and Arctic charr (Fig. 4a). However, this may not reflect species specific variance, but instead higher initial $C V_{\varepsilon}$ in the former studies. Fish were selected for these 
studies to be within certain size ranges and, therefore, the $C V_{\varepsilon}$ does not reflect the natural

340 variation of the species, but rather the abundance of fish available. Combined, these results suggest that the variance encountered in growth studies of different species of fish is similar, suggesting, that similar experimental designs are appropriate for all these species.

343 The model calculation conducted in this study show, as expected, that both the number of fish

344 in each treatment and the level of replication affect the MDD. Increasing $n$ up to 100 345 decreases the MDD considerably, while increasing $n$ over 100 has a limited effect (Fig. $3465 \mathrm{a}, \mathrm{b}, \mathrm{c})$. Increasing the level of replication from duplicates to triplicates reduces the MDD by 347 about $30 \%$. Further increases in the level of replication will reduce the MDD further, although 348 the gain in reduced MDD is progressively decreased with each increase in level of replication.

349 The MDD is of particular interests for researchers. The average expected MDD for mixed 350 model ANOVA (for statistical power of $80 \%$ ) in the experimental data analysed from the 351 different growth studies (Table 1) was $23 \%$ of the mean (range: 6-55\%). In studies published 352 in Aquaculture during the last year (Table 3), treatments in triplicate were the most common 353 (83\% of studies), with duplicates (10\%) and quadruplicates (3\%) being less common. One 354 study used six tanks per treatment. The mean number of fish in each tank in these studies was 35525.7 (range: 4-100). For triplicates, $n$ of 26 and statistical power of $80 \%$, the expected 356 minimum detectable difference is $26 \%$ when variance is average. These results suggest that in 357 most growth studies published, differences smaller than about $25 \%$ of the grand mean are not 358 reliably detected (i.e. in least $80 \%$ of trials) and half of studies will fail to detect true 359 differences under $20 \%$.

360 Researcher can take active measures to increase the resolution of statistical tests by increasing 361 the level of replication and the $n$. Furthermore, when $C V_{\varepsilon}$ and $C V_{\beta}$ are low the MDD is also 362 reduced. Both $C V_{\varepsilon}$ and $C V_{\beta}$ tend to increase as the experiments progressed (Fig. 2a,b) and this 363 was also the case in $74 \%$ of the growth studies published in Aquaculture during the last year 
364 (Table 3). However, the initial variance and final variance are positively correlated and,

365 therefore, our results suggest that it is possible to reduce the MDD further by selecting fish for 366 experiments within a narrow size range. By using stochastic models Imsland (2001) 367 suggested, that there were two main causes for size variation seen in laboratory studies with 368 turbot: (a) Individual genetical growth rate variation, this trait is stochastic in the population 369 and changes with time (stochastic growth with memory) (b) Combination of individual 370 genetical growth rate and size-related dominance hierarchies. By selecting fish within a 371 narrow size range both a) and b) above will be minimized which makes it possible to reduce 372 MDD. However, if the treatments are size specific, i.e. treatment effect depends on size, 373 selection of fish within a narrow size range may produce a bias in the results.

374 When the differences among treatments in growth studies are small, the duration of the 375 experiment is also important. As most of the growth experiments evaluated in this study 376 progressed, both $C V_{\varepsilon}$ and $C V_{\beta}$ tended to level off (Fig. 2a,b,c). If $C V_{\varepsilon}$ and $C V_{\beta}$ are stable while 377 the difference in mean size of treatment groups increases with time, statistical power will 378 increase. Furthermore, both $C V_{\varepsilon}$ and $C V_{\beta}$ are reduced as size increases (Fig. 4a,b). Therefore, 379 in order to avoid type II errors, the duration of experiments must be extended where 380 differences between effects of different treatments are small for adequate time.

381 Another possibility to increase statistical power is to include data from the entire study rather 382 than analysing only the final size of the fish. This can be done with mixed model ANOVA by 383 including time either as a categorical factor (Ling 2007), as a covariate or using repeated 384 measures ANOVA (Imsland 2001). When time is included as a covariate the growth 385 performance is compared as the slopes of the growth curves rather than the final size. 386 However, when there are large differences in the size of the fish at different times, the 387 variances may not be equal and then one of the assumptions of the ANOVA may be violated. 
Therefore, it may be necessary to use statistical procedures such as GLM in R which allows

389 data with gamma distribution or PROC MIXED in SAS where variance and covariance 390 structures can be directly modelled.

391 The results of the present study are an interesting contribution to the discussion of which is 392 the most appropriate statistical method to analyse data from growth studies. Analysing 393 published data on feed studies, Shearer (2000) suggested that ANOVA, in dose-response 394 studies, might under-estimate the critical treatment effect required to produce a maximum 395 response due to the inability of ANOVA to detect small differences. Instead he recommended 396 using regression techniques, either polynomial or logistic. However, the results of the 397 simulations performed in the present study directly contradict his conclusion. They suggest 398 that ANOVA does not necessarily underestimate the critical treatment effect. In fact, the 399 estimate of critical treatment with ANOVA most closely matched the critical value of the 400 populations. Polynomials tended to overestimate the critical treatment level by $11 \%$ on 401 average. With the logistic asymptotic function, it is difficult to decide when the maximum 402 response is reached and this will limit its usefulness. Furthermore, the logistic regression 403 procedure failed in many cases to fit the model, especially when the treatment effect was 404 small. Moreover, the advantage of using ANOVA rather than the linear and nonlinear 405 methods is that it does not presuppose the shape of the relationship between treatment and 406 effect. Therefore, we suggest that a mixed model ANOVA is the most appropriate statistical 407 method to analyse data from growth studies.

\section{4.1. Conclusions}

409 The results of this study suggest that the variance in aquaculture growth studies on different 410 species is similar and, therefore, a similar experimental design (replication level and number 411 of fish in each unit) can be employed in growth studies regardless of the species of fish. The 412 results of the study suggest that most aquaculture growth studies cannot reliably (with $80 \%$ 
413 power) detect a difference in weight that is less than $26 \%$. However, researchers can take 414 measures to reduce the minimum detectable difference by selecting fish within a narrow size 415 range for experiments. This may reduce the MDD to 5\% with adequate replication.

416 The results of the present study suggest, that in contrast to the suggestions of Baker (1986), 417 Cowey (1992) and Shearer (2000), a mixed model ANOVA is the best approach to analyse 418 growth data with graded responses and superior to non-linear models.

\section{Acknowlegdements}

420 This work was funded by the United Nation University, Fisheries Training Programme. The 421 research team at Holar University College and Maurice Ssebisubi are thanked for providing 422 their data. Parts of the paper were written while HT was a visiting scholar at the Department 423 of Environmental and Health Studies at Telemark University College, Norway. 


\section{References}

426 Araujo, P., Frøyland, L., 2007. Statistical power and analytical quantification. J. Chromotogr. $427847,305-308$.

428 Baker, D.H. 1986. Problems and pitfalls in animal experiments designed to establish dietary 429 requirements for essential nutrients. J. Nutr. 116, 2339-2349.

430 Cowey C.B.1992. Nutrition: estimating the requirements of rainbow trout. Aquaculture 100, $431 \quad 177-189$.

432 Faul, F., Erdfelder, E., Lang, A.G., Buchner, A., 2007. G*Power 3: A flexible statistical 433 power analysis program for the social, behavioral, and biomedical sciences. Behavior 434 Research Methods 39, 175-191.

435 Hurlbert, S.H. 1984. Pseudoreplication and the design of ecological field experiements. 436 Ecological Monographs 54: 187-211.

437 Imsland, A.K., Gústavsson, A., Gunnarsson, S., Foss, A., Árnason, J., Jónsson, A., 438 Smáradóttir, H., Arnarson, I., Thorarensen, H. 2008. Effects of reduced salinities on growth, 439 feed conversion efficiency and blood physiology of juvenile Atlantic halibut (Hippoglossus 440 hippoglossus L.). Aquaculture 274, 254-259.

441 Imsland, A.K., Gunnarsson, S., Roth, B., Foss, A., Le Deuff, S., Norberg, B., Thorarensen, 442 H., Helming, T. 2013. Long-term effect of photoperiod on growth, maturation and flesh 443 quality in turbot. Aquaculture 416-417; 152-160.

444 Imsland, A.K., 2001. Growth mechanisms in fishes and the use of stochastic models to 445 simulate growth and size-variation. Cand. scient. thesis in applied statistics, Dept. Math., 446 University of Bergen, $105 \mathrm{pp}$.

447 Kenward, M.G. and Roger, J.H. (1997). Small sample inference for fixed effects from 448 restricted maximum likelihood. Biometrics 53, 983-997. 
449 Kenward, M. G. and Roger, J. H. 2009. An improved approximation to the precision of fixed 450 effects from restricted maximum likelihood. Computational Statistics and Data Analysis, 53, $451 \quad 2583-2595$.

452 Kozlov, M.V., Hurlbert, S.H. 2006. Pseudoreplication, chatter, and the international nature of 453 science: A response to D. V. Tatarnikov. Journal of Fundamental Biology (Moscow) 67, 145454152.

455 Ling, E.N., Cotter, D., 2003. Statistical power in comparative aquaculture studies. 456 Aquaculture 224, 159-168.

457 Ling, E.N. 2007. Efficient analysis of growth trial data. Aquaculture research 38, 728-732.

458 Pinheiro J, Bates D, DebRoy S, Sarkar D and R Core Team (2014). _nlme: Linear and 459 Nonlinear Mixed Effects Models. R package version 3.1-117, <URL: http://CRAN.R460 project.org/package $=$ nlme $>$.

461 R Core Team (2014). R: A language and environment for statistical computing. R Foundation 462 for Statistical Computing, Vienna, Austria. URL http://www.R-project.org/.

463 Ruhonen, K 1998. Individual measurements and nested designs in aquaculture experiments: a 464 simulation study. Aquaculture 165: 149-157.

465 Schram, E., Roques, J.A.C, Abbink, W, Yokohama, Y., Spanings, T., Vries, P., Bierman, S., 466 Vis, H.v.d., Flik, G. 2012. The impact of elevated water nitrate concentration on physiology, 467 growth and feed intake of African catfish Clarias gariepinus (Burchell 1822). Aquaculture 468 Research, 1-13

469 Searcy-Bernal, R. 1994. Statistical power and aquaculture research. Aquaculture 127: 371 470388.

471 Shearer, K.D. 2000. Experimental design, statistical analysis and modelling of dietary nutrient 472 requirement studies of fish: a critical review. Aquac. Nutr. 6, 91-102. 
473 Smart, T.S., Riley, J., Edwards, P. 1998. Statistical aspects of aquaculture research: sample 474 sizes for pond experiments. Aquac. Res. 29, 373-379.

475 Sokal, R.S., Rolf, F.J. 2012. Biometry. The principles and practices of statistics in biological 476 research. W.H.Freeman and Company, New York.

477 Ssebisubi, M., 2008. Effect of water temperature and feeding level on survival, feed 478 utilization, growth and whole body composition of Oreochromis shiranus (Trewavas, 1983) 479 at different body sizes. M.Sc. thesis, Dept. of Aquaculture and Fisheries Sci., University of 480 Malawi, 158pp.

481 Tang, P.C., 1938. The power function of the analysis of variance tests with tables and 482 illustrations of their use. Sta. Res. Memor. 2, 126-149.

483 Tobin, T., Kause, A., Mäntysaari, E.A., Martin, S.A.M, Houlihan, D.F., Dobly, A., Kiessling , 484 A., Rungruangsak-Torrissen, K., Ritola, O., Ruohonen, K. 2006. Fat or lean? The quantitative 485 genetic basis for selection strategies of muscle and body composition traits in breeding 486 schemes of rainbow trout (Oncorhynchus mykiss)

487 Thorarensen, H., Gustavsson A., Mallya Y., Gunnarsson S., Arnason, J., Arnarson, I., Jonsson 488 F. A., Smaradottir, H., Zoega, T. G., Imsland, K. A., 2010. The effect of oxygen on the 489 growth and feed conversion of Atlantic halibut (Hippoglossus hippoglossus L.). Aquculture $490 \quad 309,96-102$. 
491 Table 1. Variance and power in 24 independent growth studies on fish.

\begin{tabular}{|c|c|c|c|c|c|c|c|c|c|c|}
\hline Study & Species & $\begin{array}{c}\text { Treatment } \\
\text { levels }{ }^{1}\end{array}$ & $\begin{array}{l}\text { No. of } \\
\text { tanks }^{2}\end{array}$ & $\mathrm{~N}^{3}$ & $\begin{array}{l}\text { Average final } \\
\text { body mass }(\mathrm{g})^{4}\end{array}$ & $\begin{array}{l}\text { d }(\% \text { of } \\
\text { grand } \\
\text { mean })^{5}\end{array}$ & $C V_{\varepsilon}^{6}$ & $C V_{\beta}^{\top}$ & $\begin{array}{l}\text { Observe } \\
\text { d power }^{8}\end{array}$ & $\begin{array}{l}\text { Minimum } \\
\text { detectable } \\
\text { difference at } \\
80 \% \text { power }\end{array}$ \\
\hline 1 & Halibut & 5 & 3 & 47 & 122 & 24 & 0.32 & 0.00 & 99 & 11 \\
\hline 2 & Turbot & 3 & 3 & 36 & 330.3 & 30 & 0.28 & 0.09 & 44 & 36 \\
\hline 3 & Tilapia & 3 & 6 & 16 & 11.3 & 56 & 0.37 & 0.04 & 100 & 33 \\
\hline 4 & Arctic charr & 7 & 4 & 50 & 4.7 & 30 & 0.25 & 0.07 & 100 & 22 \\
\hline 5 & Arctic charr & 7 & 4 & 39 & 10.9 & 17 & 0.28 & 0.08 & 49 & 28 \\
\hline 6 & Arctic charr & 6 & 4 & 50 & 90 & 12 & 0.21 & 0.09 & 23 & 32 \\
\hline 7 & Arctic charr & 6 & 3 & 35 & 230.8 & 11 & 0.24 & 0.04 & 34 & 21 \\
\hline 8 & Arctic charr & 6 & 3 & 132 & 672.8 & 4 & 0.15 & 0.02 & 40 & 8 \\
\hline
\end{tabular}




\begin{tabular}{|c|c|c|c|c|c|c|c|c|c|c|}
\hline 9 & Arctic charr & 6 & 3 & 64 & 1067.9 & 4 & 0.18 & 0.00 & 20 & 9 \\
\hline 10 & Arctic charr & 6 & 3 & 60 & 1437.5 & 10 & 0.17 & 0.00 & 98 & 15 \\
\hline 11 & Arctic charr & 6 & 3 & 96 & 886.7 & 17 & 0.39 & 0.06 & 55 & 27 \\
\hline 12 & Arctic charr & 16 & 3 & 30 & 2.3 & 37 & 0.26 & 0.06 & 100 & 33 \\
\hline 13 & Arctic charr & 6 & 3 & 90 & 1082.9 & 6 & 0.16 & 0.03 & 23 & 12 \\
\hline 14 & Arctic charr & 16 & 4 & 151 & 4.7 & 19 & 0.26 & 0.06 & 97 & 23 \\
\hline 15 & Atlantic cod & 5 & 3 & 13 & 800 & 18 & 0.36 & 0.00 & 41 & 31 \\
\hline 16 & Atlantic cod & 5 & 3 & 12 & 1497.3 & 13 & 0.33 & 0.00 & 60 & 6 \\
\hline 17 & Atlantic cod & 5 & 3 & 46 & 248.7 & 7 & 0.32 & 0.05 & 12 & 24 \\
\hline 18 & Atlantic cod & 6 & 3 & 15 & 791.8 & 20 & 0.35 & 0.00 & 46 & 32 \\
\hline 19 & Atlantic cod & 6 & 3 & 32 & 105.2 & 37 & 0.32 & 0.12 & 37 & 55 \\
\hline 20 & Atlantic cod & 3 & 6 & 56 & 1.9 & 16 & 0.36 & 0.07 & 38 & 17 \\
\hline 21 & Atlantic cod & 2 & 9 & 105 & 1.8 & 17 & 0.39 & 0.10 & 92 & 14 \\
\hline
\end{tabular}




\begin{tabular}{|c|c|c|c|c|c|c|c|c|c|c|}
\hline 22 & Atlantic cod & 2 & 5 & 31 & 0.23 & 13 & 0.48 & 0.11 & 29 & 28 \\
\hline 23 & Atlantic cod & 2 & 5 & 35 & 0.52 & 8 & 0.36 & 0.00 & 44 & 12 \\
\hline 24 & Atlantic cod & 2 & 5 & 14 & 0.08 & 13 & 0.56 & 0.00 & 13 & 31 \\
\hline
\end{tabular}

$492{ }^{\mathrm{T}}$ Number of treatments tested in the experiment.

$493{ }^{2}$ Number of tanks tested for each treatment.

$494{ }^{3}$ Number of fish in each tank.

$495{ }^{4}$ Mean body-mass of fish $(\mathrm{g})$ in a study.

$496{ }^{5}$ Maximum difference between treatments means ((\% of grand mean).

$497{ }^{6}$ Error coefficient of variation $\left(C V_{\varepsilon}\right)$.

$498{ }^{7}$ Coefficient of variation for tanks within treatment $\left(C V_{\beta}\right)$.

$499{ }^{8}$ Retrospective power (\%) at the end of studies.

$500{ }^{9}$ Effect size (\% of grand mean) at $80 \%$ power.

501 Data from: 1-Thorarensen et al. (2010); 2-Le Deuff et al.(2010); 3-Ssebisubi, (2008);.4-14-Sigurgeirsson et al., unpublished ; 15-Sigurgeirsson 502 and Árnason, unpublished; 16-21-Árnason et al., unpublished; 22-24-Edelsparre and Pálsson, unpublished. 
Table 2. Summary of analyses from simulation studies on data sampled from artificial populations, two with graded responses (Res11\% and

Res45\%) and one population with no response to treatment (Res0\%). Randomized normally distributed data with equal variances was generated

$100 \%$ for all artificial populations and the maximum response was $100 \%$.

\begin{tabular}{|c|c|c|c|c|c|c|c|c|c|}
\hline & \multicolumn{3}{|c|}{ ANOVA } & \multicolumn{3}{|c|}{ Second order polynomial } & Three & $\begin{array}{l}\text { parameter } \\
\text { regression }\end{array}$ & gistic \\
\hline Mean critical treatment & 99.7 & 95.0 & 92.3 & 110.7 & 108.8 & 96.5 & 101.5 & 97.0 & \\
\hline Median critical treatment (\%) & 100 & 95 & 92 & 111 & 108 & 98 & 101 & 92 & - \\
\hline Mean maximum response & 100 & 101 & 100 & 103 & 102 & 105 & 96.8 & 97 & \\
\hline
\end{tabular}


Mean effect size as \% of

18.3

13.1

9.0

grand mean (95\% range)

$(10.2-26.9) \quad(8.8-17.5) \quad(5.8-12.0)$

Mean square residual

deviation $^{3}$

$8.4 \quad 12$

$2.0 \quad 23.4$

10.2

16.5

8.9

3.8

Proportion of analyses

showing a significant effect

$100 \%$

$36 \%$

$1 \%$

$100 \%$

$51 \%$

$5 \%$

of treatment

Analysis producing an error

message

512 The treatment effect required to give maximum response

$513{ }^{2}$ Estimated maximum effect.

$514{ }^{3}$ The mean square residual deviation between predicted responses and population responses. 
515 Table 3. A summary of variability of final body mass and experimental design in 29 growth

516 studies of 24 species of fish published in 2013 and 2014 in Aquaculture. The $C V_{\beta}$ were

517 estimated based on reported standard errors and levels of replication in studies where simple

518 ANOVA was used for statistical analysis.

\begin{tabular}{lccc}
\hline & Mean & Range & Mean \\
& & & factorial \\
& & & increase $^{2}$ \\
\hline$C V_{\varepsilon}(\%)^{1}$ & 27.9 & $23-36$ & 1.78 \\
$C V_{\beta}(\%)$ & 4.9 & $0-49$ & 1.75 \\
Level of replication (rearing units / treatment) & 3 & $2-6$ & \\
Number of fish in each rearing unit & 25.7 & $4-100$ & \\
\hline
\end{tabular}

$519{ }^{\mathrm{I}}$ Information on $C V_{\varepsilon}$ was only available in 4 studies.

$520{ }^{2}$ Final divided by the initial $C V_{\varepsilon}$ and $C V_{\beta}$. 
Figure captions

523

524

525

526
Figure 1. The three populations used in the model simulations: Res $45 \%$ where the minimum treatment gave a response that was $45 \%$ less than the maximum; Res $11 \%$ where the minimum treatment gave a response that was $11 \%$ less than the maximum; and Res $0 \%$ where treatment had no effect on response. The units for treatment and response are shown as percentages. For Res $11 \%$ and Res $45 \%$, a treatment level of $100 \%$ will produce a $100 \%$ response.

Figure 2: Development of $C V_{\varepsilon}$ with increasing body mass in experiments on (a) tilapia, Atlantic halibut and turbot, (b) Atlantic cod and (c) Arctic charr. (The different lines represent separate studies). The increase in body mass is shown as factorial increase (mean body-mass / mean initial body-mass).

Figure 3: Development of $C V_{\beta}$ with increasing body mass in experiments on (a) tilapia, Atlantic halibut, and turbot, (b) Atlantic cod and (c) Arctic charr. (The different lines represent separate studies). The increase in body mass is shown as factorial increase (mean body-mass / mean initial body-mass).

Figure 4: Coefficients of variation in growth studies of Atlantic cod and Arctic charr at different final mean body mass. a) $C V_{\varepsilon}$ and mean final body-mass. The intercepts for the two species were significantly different $(\mathrm{p}<0.0001)$ while the slopes of the regression lines for the two species were not significantly different. The regression lines (interrupted for the Atlantic cod, continuous for Arctic charr) with a common slope was $C V_{\beta}=$ Intercept $-0.006 \times$ body mass with the intercepts being 25.7 and 40.6 for the Arctic charr and Atlantic cod respectively. b) $C V_{\beta}$ and mean final body-mass. Neither slopes nor intercepts were significantly different. The common regression line was: $C V_{\beta}=6.67-0.005 \times$ body-mass $\left(R^{2}\right.$ : $0.38)$.

Figure 5: Minimum detectable difference (MDD), shown as \% of the grand mean in growth studies with five treatments levels when statistical power is $80 \%$. a) Mean $C V_{\varepsilon}$ and mean $C V_{\beta}$. b) High $C V_{\varepsilon}$ and high $C V_{\beta}$. c) Low $C V_{\varepsilon}$ and low $C V_{\beta}$. 
550 Figure 1.

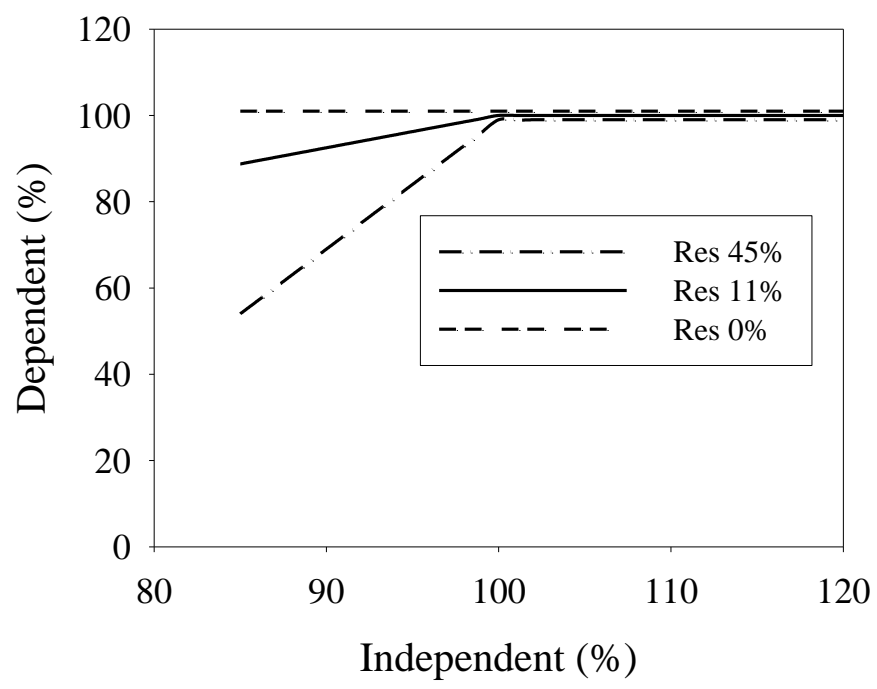

551 
Figure 2.
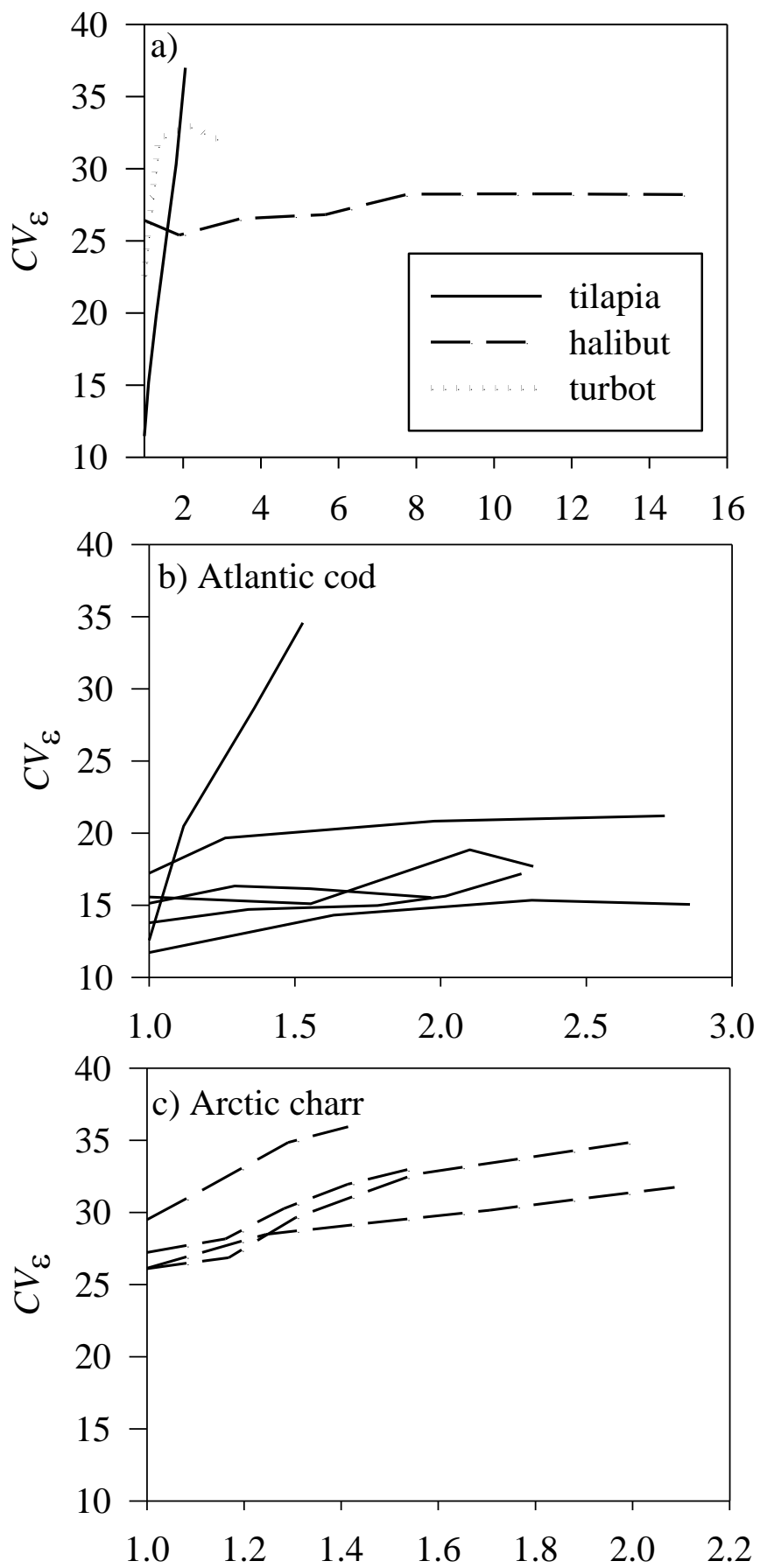

554

Factorial increase in body mass 
Figure 3.
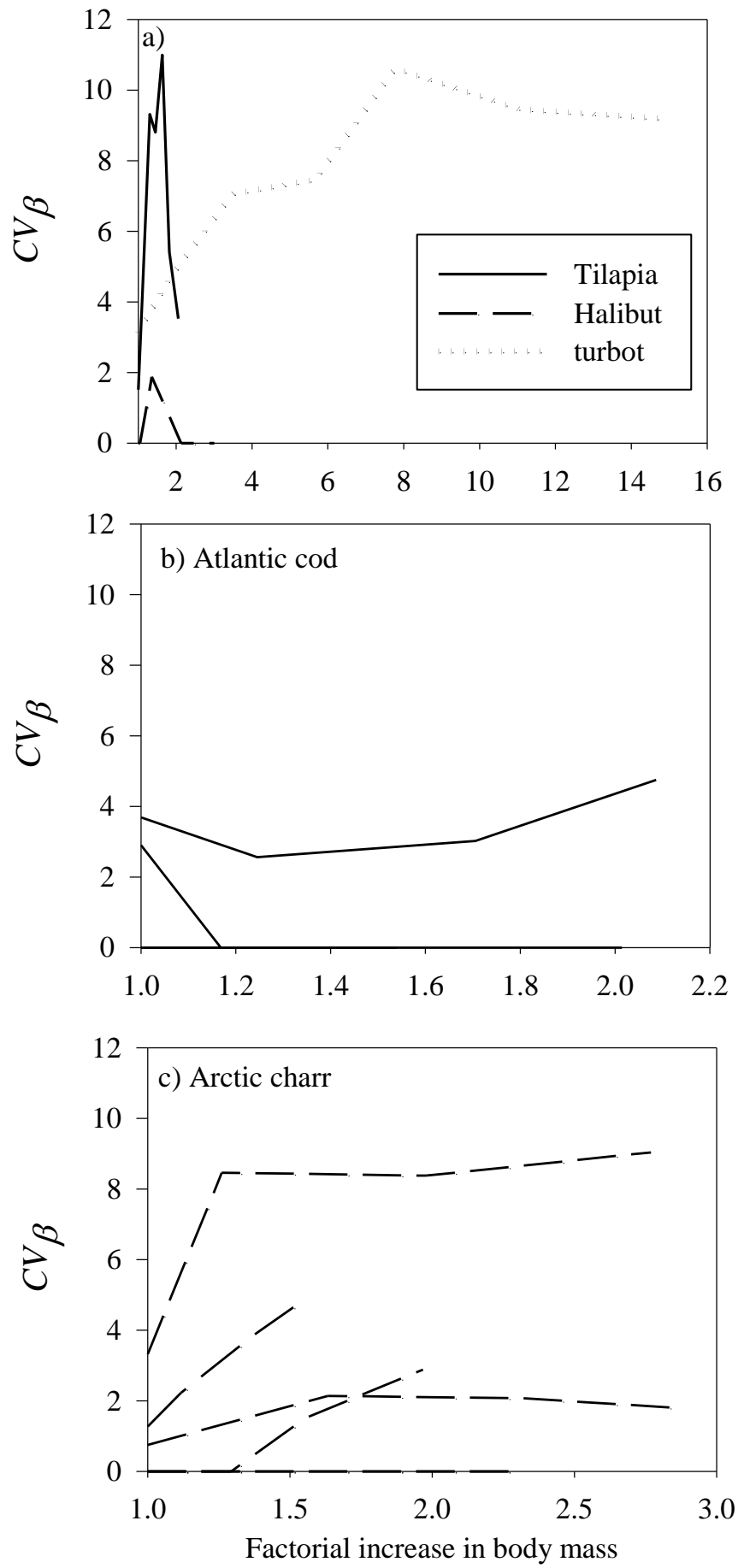
Figure 4.
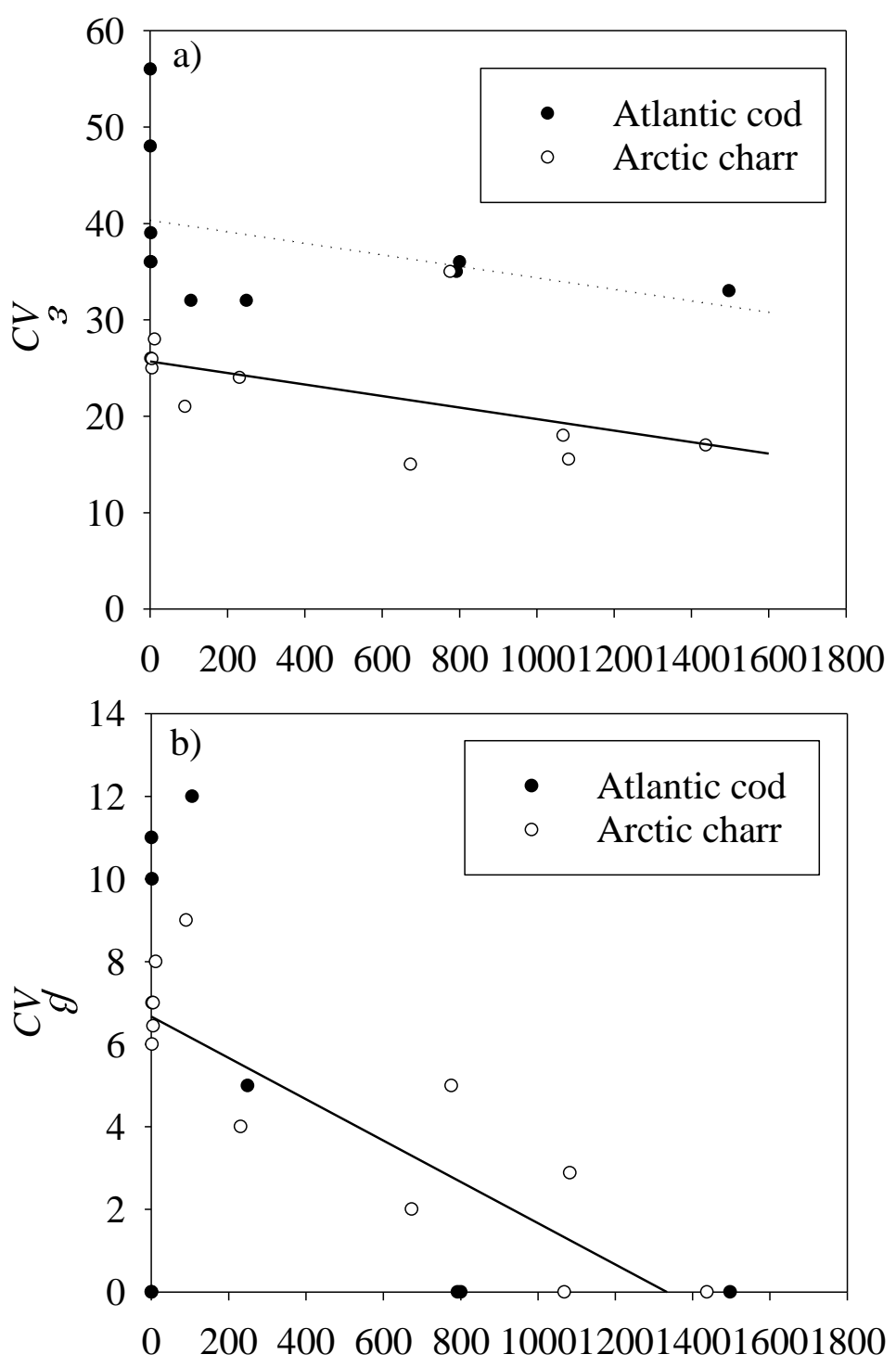

Final mean body mass (g) 
561 Figure 5.
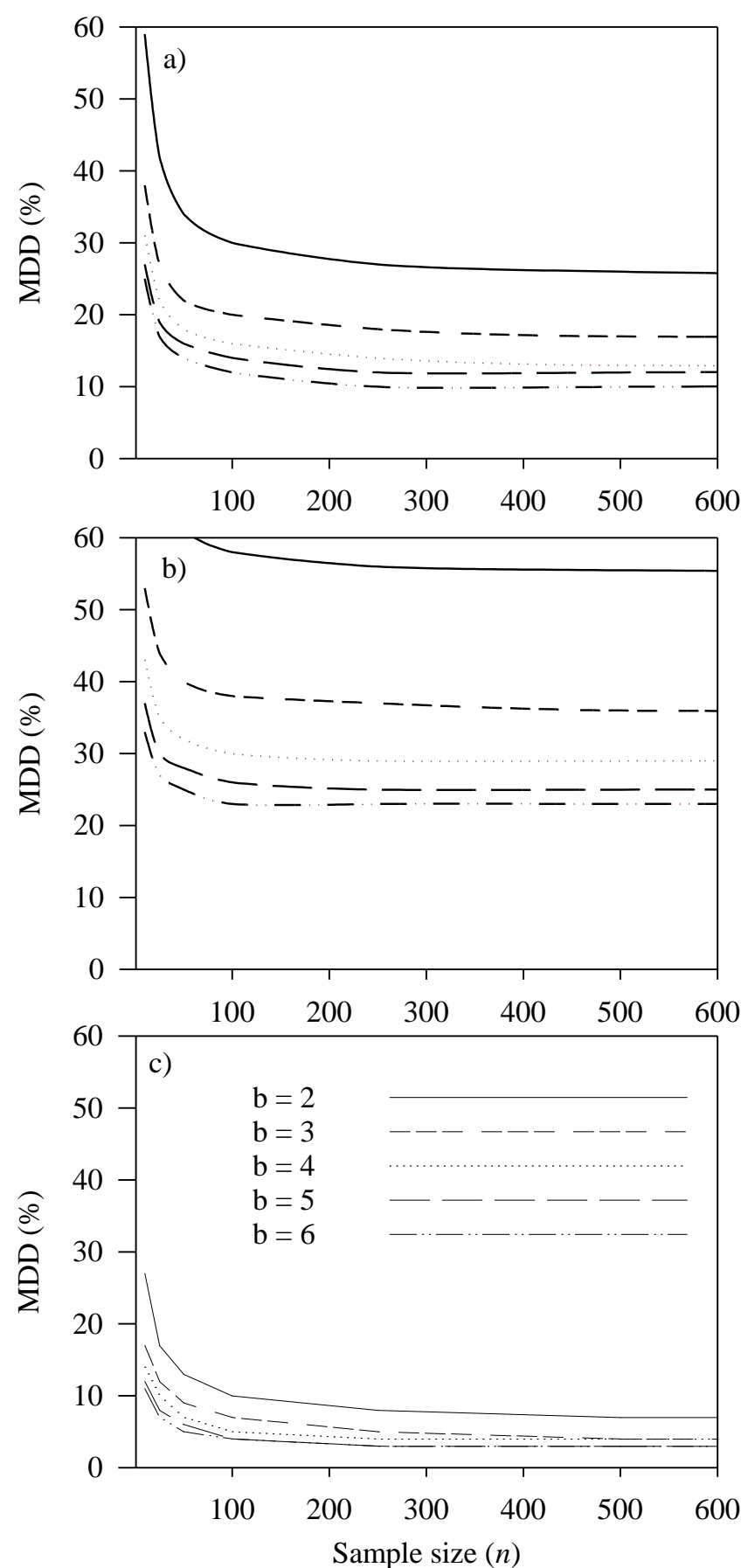

562

Sample size $(n)$ 